\title{
Importance of material parameters and strain energy function on the wall stresses in the left ventricle
}

\section{Sareh BEHDADFAR}

Ecole Nationale Supérieure des Mines, CIS-EMSE, Institut national de la santé et de la recherche médicale, INSERM:UMR1059, SAINBIOSE, F-42023 Saint-Etienne, France

behdadfar@emse.fr $\quad+33632247449$

Laurent NAVARRO

Ecole Nationale Supérieure des Mines, CIS-EMSE, Institut national de la santé et de la recherche médicale, INSERM:UMR1059, SAINBIOSE, F-42023 Saint-Etienne, France

navarro@emse.fr $\quad+33671196331$

\section{Joakim SUNDNES}

Computational Cardiac Modeling department, Simula research laboratory, Martin Linges vei 25, 1364 Fornebu, Norway

sundnes@ simula.no $\quad+4797722924$

Molly M. MALECKAR

Allen Institute, 615 Westlake Ave N, Seattle, WA 98109, USA-Simula research laboratory, Martin Linges vei 25, 1364 Fornebu, Norway mmaleck@ simula.no

\section{Stéphane AVRIL}

Ecole Nationale Supérieure des Mines, CIS-EMSE, Institut national de la santé et de la recherche médicale, INSERM:UMR1059, SAINBIOSE, F-42023 Saint-Etienne, France avril@emse.fr $+33603755029$ 


\title{
Importance of material parameters and strain energy function on the wall stresses in the left ventricle
}

\begin{abstract}
Patient-specific estimates of the stress distribution in the left ventricles (LV) may have important applications for therapy planning, but computing the stress generally requires knowledge of the material behaviour. The passive stress-strain relation of myocardial tissue has been characterized by a number of models, but material parameters (MPs) remain difficult to estimate. The aim of this study is to implement a zero-pressure algorithm to reconstruct numerically the stress distribution in the LV without precise knowledge of MPs. We investigate the sensitivity of the stress distribution to variations in the different sets of constitutive parameters. We show that the sensitivity of the LV stresses to MPs can be marginal for an isotropic constitutive model. However, when using a transversely isotropic exponential strain energy function, the LV stresses become sensitive to MPs, especially to the linear elastic coefficient before the exponential function. This indicates that in-vivo identification efforts should focus mostly on this MP for the development of patient-specific finite-element analysis.
\end{abstract}

Keywords: cardiac mechanics; stress sensitivity analysis; inverse method;

\section{Introduction}

Nowadays, there is a growing interest in patient-specific finite-element (FE) stress analysis in many soft tissues (Wang et al. 2002-2013). It is postulated that in normal homeostatic conditions, tissues adapt, grow or remodel themselves in order to regulate the stresses (Humphrey 2006, Graham et al. 1968). Consequently, the stress distribution may provide important indications about the mechanobiological function and metabolism of the tissue. As far as the left ventricle (LV) is concerned, geometrical adaptation may occur after myocardial infarction and also after myocardial injection when this treatment is employed to avoid possible risks of heart failure (Kichula et al. 2014). In these different situations of adaptation, reconstructing the stress distribution may be very useful. 
Researchers mostly focused their attention on the reconstruction of three dimensional strain maps throughout a cardiac cycle (Tustison et al. 2006; Spottiswoode et al. 2007) using medical imaging modalities such as gated MRI or ultrasound (US). To assess the wall stress distribution from these strain maps, it is necessary to know the constitutive material parameters (MPs) and initial stresses in the tissue.

Characterizing the MPs involves several difficulties. The MPs may be obtained using in-vitro experiments carried out on myocardial tissue collected on cadavers (Gee et al. 2010). However, this method does not yield patient-specific MPs that can be used for clinical applications. Another approach is to calibrate a model against clinical data (Krishnamurthy et al., 2013, Klotz et al., 2006, Raghavan et al., 2006). One has to define a cost function to minimize the differences between data and the model. It may be difficult, though, to ensure a unique solution to the minimization problem in case of inadequate clinical data.

However, stress analysis does not systematically require a precise knowledge of MPs, as Miller and Lu (2013) and Joldes et al. (2016) have shown for arteries and brain tissue for which the numerical simulations are only weakly sensitive to the MPs. Based on this, Lu et al. (2008) developed an inverse analysis method to reconstruct stress distributions in thin-walled biological organs. The point of their approach is that the stress field in a statically determinate problem only depends on the boundary conditions and on the geometry but not on the MPs. They showed an example of successful application to anaortic aneurysm but they did not discuss possible applications on thickwalled geometries such as the LV.

The hypothesis of the present study is that this approach of stress estimation without knowing the MPs could be extended to the reconstruction of the stress distribution in the LV. We will assess the validity of this hypothesis first for a passive LV, both for a 
simple isotropic material model and a more complex transversely isotropic model regarded as a realistic representation of passive myocardial tissue (Guccione et al., 1995). Finally, we assess the hypothesis for a material model with active contraction, for reconstructing the systolic LV stress distribution. After giving the details for reconstructing end-diastolic (ED) and end-systolic (ES) stress distributions based on the proposed approach, we draw conclusions for potential future studies.

\section{Materials and Methods}

\subsection{Geometrical Model}

The LV of a healthy volunteer was segmented from a 4D US scan using the VTK library (Hansegård et al. 2009). The mesh at ED was reconstructed with 8-noded linear brick elements $(155,172$ nodes and 141,405 elements, after performing a convergence study, see Figure 1(a)). The nodes of the base were constrained to remain coplanar during the deformations. A local curvilinear coordinate system aligned along the fiber direction was considered (Bovendeerd et al. 1994). Fiber directions as proposed in the literature (Guccione et al., 1995) varied from $-70^{\circ}$ at the epicardial surface to $60^{\circ}$ at the endocardial surface, where $0^{\circ}$ was the circumferential direction (Figure 2). At every element across the wall of the LV, we defined a local orthonormal basis denoted $\left(\overrightarrow{\boldsymbol{e}}_{\boldsymbol{n}}, \overrightarrow{\boldsymbol{e}}_{\boldsymbol{s}}, \overrightarrow{\boldsymbol{e}}_{\boldsymbol{f}}\right)$, where $\overrightarrow{\boldsymbol{e}}_{\boldsymbol{f}}$ was aligned along the local fiber direction in the reference configuration.

\subsection{Method for Numerical Stress Reconstruction at ED}

The in-vivo heart is never unloaded, and the ED geometry extracted from US images corresponded to a non-zero cavity pressure. The unloaded geometry was reconstructed using the approach of Riveros et al. (2013); we refer to this method as the zero-pressure algorithm (Trabelsi et al. 2015). More specifically, we iteratively updated the unloaded 
geometry and computed its deformation upon pressurization (endocardial surface loaded with a $2 \mathrm{kPa}$ pressure) until the deformed geometry matched the ED geometry (tolerance $\leq 0.028 \mathrm{~mm}$ ) obtained from US images. The deviation between the deformed geometry and the US-derived ED geometry was obtained by calculating the absolute maximum nodal distance as illustrated in Figure 3.

After deriving the unloaded geometry, the Cauchy stresses at ED were deduced such as:

$$
\boldsymbol{\sigma}_{\text {passive }}=J^{-1} \boldsymbol{F} \frac{\partial W}{\partial \boldsymbol{E}} \boldsymbol{F}^{T}
$$

where $W$ is the strain energy function, $\boldsymbol{F}$ is the deformation gradient, $J=\operatorname{det}(\boldsymbol{F})$ is the determinant of the deformation gradient, and $\boldsymbol{E}$ is the Green-Lagrange strain tensor.

In the literature, different strain energy functions have been proposed for the passive behaviour of cardiac tissue, as in Holzapfel et al. (2009), Guccione et al. (1995) and Usyk et al. (2000). Guccione et al. (1995) proposed a transversely isotropic Fung model and Usyk et al. (2000) proposed an orthotropic Fung model. In order to investigate the impact of the strain energy function on the stress reconstruction, we used first a simple Mooney-Rivlin strain energy function as previously used by Marchesseau et al., (2013):

$$
\mathrm{W}=\mathrm{c}_{1}\left(\overline{\mathrm{I}}_{1}-3\right)+\mathrm{c}_{2}\left(\overline{\mathrm{I}}_{2}-3\right)+\mathrm{K}(\mathrm{J}-1)^{2}
$$

where $c_{1}$ and $c_{2}$ are MPs, $\overline{\mathrm{I}}_{1}$ and $\overline{\mathrm{I}}_{2}$ are invariants of the right Cauchy-Green strain tensor and $K=2 / d_{1}$ is the bulk modulus, $d_{1}$ being the compressibility term. Wang et al. (2013) showed that the transversely isotropic and orthotropic Fung models provide similar results. Therefore, we used the Fung-type strain energy function developed by Guccione et al. (1995): 


$$
\begin{aligned}
& W=\frac{1}{2} c(\exp (\bar{Q})-1)+\mathrm{K}(\mathrm{J}-1)^{2}, \\
& \quad \bar{Q}=b_{3} \bar{E}_{f f}^{2}+b_{2}\left(\bar{E}_{s S}^{2}+\bar{E}_{n n}^{2}+\bar{E}_{s n}^{2}\right)+b_{1}\left(2 \bar{E}_{f S}^{2}+2 \bar{E}_{f n}^{2}\right),
\end{aligned}
$$

where $W$ is a function of six independent components of the isochoric Green-Lagrange strain tensor $\bar{E}_{i j}$ in fiber (f), sheet (s) and sheet-normal (n) directions, and $c$ and $b_{1-3}$ are the four MP values.

Finally, we compared our approach to the method of Joldes et al. (2016) for stress reconstruction, which consists of assigning very stiff linear elastic material properties (10 $\mathrm{MPa}$ and 0.49 as elastic modulus and Poisson's ratio) to the LV in its actual geometry at ED.

\subsection{Method for Numerical Stress Reconstruction at ES}

The Cauchy stress tensor at ES was defined as:

$$
\sigma_{\text {total }}=\sigma_{\text {active }}+\sigma_{\text {passive }}
$$

where $\sigma_{\text {active }}$ is the contribution of myocyte contraction.

We denote by $\boldsymbol{R}_{\boldsymbol{f}}$ the matrix of the covariant transformation from the global orthonormal to the local basis:

$$
\boldsymbol{\sigma}_{\text {active }}=\mathrm{J}^{-1} \mathbf{F} \mathbf{R}_{\mathbf{f}}{ }^{-1}\left[\begin{array}{ccc}
0 & 0 & 0 \\
0 & 0 & 0 \\
0 & 0 & \mathrm{a}_{\mathrm{f}}
\end{array}\right] \mathbf{R}_{\mathbf{f}} \mathbf{F}^{\mathbf{T}}
$$

where $a_{f}$ is the active tension developed by the fibers.

In physiological models, $a_{f}$ is often computed by solving systems of ordinary differential equations describing cell electrophysiology and contraction (Nash et al. 2004). Here, for the sake of simplification, we assigned a predefined value to $a_{f}$ equal 
to $135 \mathrm{kPa}$ as explained in Dorri et al. 2006 and Walker et al. 2005. In this condition, the only variations will be due to the MPs and not the active stress value. We performed FE analysis from ED to ES and estimated the induced Cauchy stress for different sets of MPs explained in the next subsection.

As the reference FE model was reconstructed at ED from US, we performed a forward simulation to obtain the reference geometry at ES. This computation was the combination of systolic cavity pressure (11.24 $\mathrm{kPa}$ at ES) and the prestress of $135 \mathrm{kPa}$ applied along the fiber directions with the following MP values for the Mooney-Rivlin strain energy function: $c_{1}=0.0176, c_{2}=0.0188$ and $d_{1}=0.1079$ in $\mathrm{MPa}$.

\subsection{Statistical Analysis}

Several FE analyses (40) were performed by drawing randomly different MP sets with uniform distribution within the range of values of Marchesseau et al. (2013) in Table 1. For each analysis, a random sample was drawn for each parameter $\left(c_{1}, c_{2}, d_{1}\right)$ to employ the zero-pressure algorithm with diastolic cavity pressure equal to $2 \mathrm{kPa}$. For the Guccione strain energy function, we collected 12 MPs obtained from literature (Table 2) and performed $12 \mathrm{FE}$ analyses with the zero-pressure algorithm $(2 \mathrm{kPa})$. In order to avoid the rigid body movements, all the nodes of the basal surface were constrained to remain coplanar during the deformation. The diastolic and systolic statistical analyses consist in calculating the mean and the standard deviation (SD) of all FE stress results from different MP sets.

FE analyses introduced in Section 2.2 and Section 2.3 were performed with the Abaqus ${ }^{\circledR}$ software on a local cluster (8-core Intel Xeon-E5530 2.4-GHz, 24-Go RAM). 


\section{Results}

The zero-pressure algorithm was applied with 40 samples of MPs for the MooneyRivlin model at ED. The maximum nodal distance observed between the reference image data and the zero-pressure geometries (ZPGs) after loading was $0.027 \pm 0.001$ $\mathrm{mm}$. The stress and strain results for the Mooney-Rivlin model are shown as colormaps in Figure 4. The Mooney-Rivlin stress values are also reported in Table 3 for different paths throughout the wall thickness (refer to Figure 1(b)). The circumferential stresses show larger values where the myocardial fibers are aligned in this direction. For all isotropic stress values, the SD is one to two orders less compared to the mean stress. In addition, the results of $99 \%$ of total LV elements vary less than $2 \%$ in terms of maximum-principal Cauchy stresses.

The stress reconstruction obtained with the approach of Joldes et al. (2016), using rigid material properties and linear infinitesimal resolution, is shown in Figure 5. It is consistent with the Mooney-Rivlin case.

The fiber stress and strain results for the Guccione model are shown as colormaps in Figure 4 as well. The trends for the variations of sheet and normal to sheet stresses are similar to the ones of fiber stresses (results not presented). The results with the Guccione material model are reported in Table 2, showing larger variations in fiber stresses than the Mooney-Rivlin model (Figure 4). The mean value for maximum distance between the reference image-based geometry and the ZPGs geometry after loading is $0.0281 \pm 8 \mathrm{e}-04 \mathrm{~mm}$. The correlation coefficients between MPs and the fiber stresses obtained from FE analyses are shown in Table 4. There is a correlation of approximately 0.8 at ED between the linear coefficient and fiber stresses (Table 4). There is only a moderate correlation between the exponential coefficients and the fiber 
and sheet stresses. Actually, the variation of stresses is fairly low for a number of sets of MPs.

The simulated reference contraction (Figure 6) produced an ejection fraction of $33.83 \%$, a wall thickness change of $18.7 \%$ and Von-Mises stresses in the range of $100-150 \mathrm{kPa}$, which were previously observed in (Dorri et al. 2006; Hunter et al.1998).

Stress maps reconstructed in the equatorial plane (Mooney-Rivlin passive model) are displayed in Figure 7, while the stress values are reported in Table 5, for the transmural paths in Figure 1(b). Here, it can be noticed that the Von-Mises stress increases from epicardium to endocardium. For all stress values, the SD is one order of magnitude lower than the mean value.

\section{Discussion}

Reconstructing the stress distribution in the LV using the zero-pressure algorithm proposed by Riveros et al. (2013), we have shown that the LV stresses can be reconstructed relatively independently of MPs.

This result is significant as in normal homeostatic conditions, tissues adapt, grow or remodel themselves in order to regulate the stresses (Humphrey 2006, Graham et al. 1968). As far as the LV is concerned, geometrical adaptation may occur after myocardial infarction and also after myocardial injection when this treatment is employed to avoid possible risks of heart failure (Kichula et al. 2014). In these different situations of adaptation, reconstructing the stress distribution using the Laplace law is not accurate enough (Huisman et al. 1980; Yin 1985). In addition, it is interesting to have a patient-specific method fairly dependent on all the sets of MPs for every patient. 
We varied the MPs either by drawing them randomly or by testing different sets of values available in the literature. However, none of the tested values were the actual MPs of our subject's myocardium. The goal was only to achieve different virtual experiments in order to emphasize that knowing all the MPs of a given subject is not required to reconstruct the $\mathrm{LV}$ stress distribution.

In our analysis, we performed some of the stress reconstructions using an isotropic strain energy function. Although it is well known that the myocardium is transversely isotropic, we showed that the reconstructed stress obtained with the isotropic model is a rather good approximation of the same reconstructed stress obtained with a transversely isotropic model. The low ejection fraction in our FE analysis is the result of this choice of material model which reduces the wall thickening in circumferential direction (Dorri et al. 2006).

Results in Figure 4 and Table 3 show a very low correlation between passive stresses and MPs in case of isotropy. This is consistent with the results obtained on other soft tissues by Miller and Lu (2013). They showed that MPs have a marginal impact on the stress distribution of inflated geometries. In that case, stress distributions can be reconstructed using a simple linear infinitesimal approach as introduced by Joldes et al. (2016).

With the commonly used Guccione model, which accounts for transverse isotropy, among all the MPs, only variations of the $c$ as the linear and $b_{2}$ coefficient parameters had significant impact on the reconstructed stress distributions. We can even see in Table 3 that the calculated fiber and sheet stresses are strongly coupled to $c$. The dependence between the reconstructed stress distributions and $c$ points out the scaling role of the linear coefficient in Fung-like strain energy functions. This also indicates 
that the stress can be reconstructed fairly independently of the exponential coefficients of the Guccione model. This is also a significant result as no efficient method exists for the moment to derive non-invasively and patient-specifically all the parameters of the Guccione model in vivo.

We also reconstructed stresses at ES with a combination of passive and active contributions. The active contribution mostly depends on $a_{f}$ and the passive contribution depends on the deformation from ED to ES, which takes the tissue from a state of circumferential tension to compression. We showed eventually in Figure 7 that passive MPs marginally impact the reconstructed stress at ES (SD less than $10 \%$ of the mean stress values), but this is induced by the small level of passive stresses compared to active stresses at ES.

\section{Limitations and future work}

The objective of this paper was to be able to reconstruct numerically the stress distribution in the LV without knowing the MPs of myocardial tissue. While promising, there are several limitations to the approach.

The active contraction model applied for the ES data is a simplification compared with the state-of-the-art models (Nash et al. 2004 and Walker et al. 2005). Another limitation is that we did not study the effect of fiber orientations on the stress reconstructions (we used always the same orientations in all our analyses) which is an open question. Palit et al. (2015) and Wang et al. (2013) showed that fibers have a significant effect on ED stress distribution and on pressure-volume relationship. Eriksson et al. (2013) showed that the heterogeneous structure of fibers also play an important role in systolic stress results. However, due to the form of the Guccione strain energy at ED, we expect that 
fiber orientations have a similar effect on the stresses as varying the exponential coefficients. We showed that this effect was marginal. Another limitation to be mentioned is that the US images were captured from the basal part and other tissues such as valves, papillary muscles and irregularities were removed. The presence of all these features may affect the local distribution of stresses as the movement of the human heart base is significant compared to the apex movement (Palit et al. 2017). This means that most of the conclusions of this study would not hold at a local scale where these features would be resolved.

\section{Conclusion}

In this study, we showed that it is possible to reconstruct numerically the stress distribution in the LV without precise knowledge of MPs of myocardial tissue. However a patient-specific geometry of the LV is always required, which highlights the importance of patient-specific FE model morphology over the MPs in cardiac modelling as mentioned in (Palit et al., 2017) and (Palit et al., 2015)Only the linear elastic coefficient of the Guccione strain energy function may be required to have an accurate stress reconstruction. This implies that the effort should focus mostly on the identification of this MP for patient-specific stress reconstructions. It is also important to continue exploring more realistic models for ES, to determine whether the same relations hold.

\section{References}

Augenstein KF, Cowan BR., LeGrice IJ, Nielsen PM,Young AA. 2005. Method and apparatus for soft tissue material parameter estimation using tissue tagged magnetic resonance imaging Journal of Biomechanical Engineering, 127(1) $: 148-157$. 
Bovendeerd PH, Huyghe JM, Arts T, Campen DHV, Reneman RS. 1994. Influence of endocardial-epicardial crossover of muscle fibers on left ventricular wall mechanics. Journal of Biomechanics., 27, pp.941-951.

Dorri F, Niederer PF, Lunkenheimer PP. 2006. A finite element model of the human left ventricular systole. Computer Methods in Biomechanics and Biomedical Engineering, 9(5):319-341.

Eriksson TSE, Prassl AJ, Plank G, Holzapfel GA. 2013. Influence of myocardial fiber/sheet orientations on left ventricular mechanical contraction. Mathematics and Mechanics of Solids, 18, 592-606.

Gee MW, Förster C, Wall WA. 2010. A computational strategy for prestressing patientspecific biomechanical problems under finite deformation. International Journal for Numerical Methods in Biomedical Engineering, 26(1) :52-72.

Graham TP, Covell JW, Sonnenblick EH, Ross JJR, Braunwald E. 1968. Control of myocardial oxygen consumption: relative influence of contractile state and tension development. . Journal of Clinical Investigation, 47(2) :375.

Guccioné JM, Costa KD,McCulloch AD. 1995. Finite element stress analysis of left ventricular mechanics in the beating dog heart. Journal of Biomechanics, 28(10), 1167-1177.

Guccioné JM, McCulloch AD, Waldman LK. 1991. Passive material properties of intact ventricular myocardium determined from a cylindrical model. Journal of Biomechanical Engineering, 113(1) :42-55.

Hansegård J, Urheim S, Lunde K, Malm S, Rabben SI. 2009. Semi-automated quantification of left ventricular volumes and ejection fraction by real-time three-dimensional echocardiography. Cardiovascular Ultrasound, 7(18):379--84.

Holzapfel G, Ogden RW. 2009. Constitutive modelling of passive myocardium: a structurally based framework for material characterization. Philosophical Transactions of the Royal Society of london A: Mathematical, Physical and Engineering Sciences, 367(1902):3445--3475.

Huisman RM, Sipkema P, Westerhof N, Elzinga G. 1980. Comparison of models used to calculate left ventricular wall force., Comparison of models 18:133-144.

Humphrey JD. 2006. Towards a Theory of Vascular Growth and Remodelling (pp. 315). Springer Berlin Heidelberg.

Hunter PJ, McCulloch AD, Keurs HE. 1998. Modelling the mechanical properties of cardiac muscle. Progress in biophysics and molecular biology , 69, pp. 289-331. 
Joldes GR, Miller K, Wittek A, Doyle B. 2016. A simple, effective and clinically applicable method to compute abdominal aortic aneurysm wall stress. Journal of the Mechanical Behavior of Biomedical Materials , 58 :139-148.

Keldermann RH, Nash MP, Gelderblom H, Wang VY, Panfilov AV. 2010. Electromechanical wavebreak in a model of the human left ventricle. American Journal Physiology-Heart and Circulatory Physiology, 299(1) :H134-H143.

Kichula ET, Wang H, Dorsey SM, Szczesny SE, Elliott DM, Burdick JA, Wenk, JF. 2014. Experimental and computational investigation of altered mechanical properties in myocardium after hydrogel injection. Annals of Biomedical Engineering, 42(7), 1546-1556.

Klotz S, Hay I, Zhang G, Maurer M, Wang J, Burkhoff D. 2006. Development of heart failure in chronic hypertensive Dahl rats focus on heart failure with preserved ejection fraction. Hypertension, 47(5), 901-911.

Krishnamurthy A, Villongco CT, Belezzuoli E, Kerckhoffs RC and et al. 2013. Patientspecific models of cardiac biomechanics. Journal of Computational Physics, 244, 4-21.

Land S, Niederer SA, Smith NP. 2012. Efficient computational methods for strongly coupled cardiac electromechanics. IEEE Transactions on Biomedical Engineering, 59(5) :1219-1228

Lu J, Zhou X, Raghavan ML. 2008. Inverse method of stress analysis for cerebral aneurysms. Biomechanics and Modeling in Mechanobiology, 7(6), 477-486.

Marchesseau S, Delingette H, Sermesant M, Ayache N. 2013. Fast parameter calibration of a cardiac electromechanical model from medical images based on the unscented transform. Biomechanics and Modeling in Mechanobiology, 12(4), 815-831.

Miller K, Lu J. 2013. On the prospect of patient-specific biomechanics without patientspecific properties of tissues. Journal of the Mechanical Behavior of Biomedical Materials, 27, 154-166.

Nash MP, Panfilov AV. 2004. Electromechanical model of excitable tissue to study reentrant cardiac arrhythmias. Progress in biophysics and molecular biology, $85(2), 501-522$.

Okamoto R, Moulton MJ, Peterson S, Li D, Pasque M, Guccioné, J. 2000. Epicardial suction: a new approach to mechanical testing of the passive ventricular wall. Journal of Biomechanical Engineering, 122(5):479-487. 
Omens JH, May KD, McCulloch AD. 1991. Transmural distribution of 3D strain in the isolated arrested canine left ventricle. American Journal Physiology-Heart and Circulatory Physiology, 261(3), H918-H928.

Omens J, MacKenna D, McCulloch A. 1993. Measurement of strain and analysis of stress in resting rat left ventricular myocardium. Journal of Biomechanics, 26(6) :665-676.

Palit A, Bhudia SK, Arvanitis TN, Turley GA, Williams MA. 2015. Computational modelling of left-ventricular diastolic mechanics: Effect of fibre orientation and right-ventricle topology. Journal of Biomechanics, 48, 604-612.

Palit A, Franciosa P, Bhudia SK, Arvanitis TN, Turley GA, Williams MA. 2017. Passive diastolic modelling of human ventricles: Effects of base movement and geometrical heterogeneity. Journal of Biomechanics, 52, 95-105.

Raghavan ML, Baoshun MA, Filinger MF. 2006. Non-invasive determination of zeropressure geometry of arterial aneurysms. Annals of Biomedical Engineering . 34(9):1414-1419.

Riveros F, Chandra S, Finol, EA, Gasser, TC, Rodriguez, JF. 2013. A pull-back algorithm to determine the unloaded vascular geometry in anisotropic hyperelastic AAA passive mechanics. Annals of Biomedical Engineering, 41(4), 694-708.

Spottiswoode BS, Zhong X, Hess AT, Epstein FH and et al. 2007. Tracking myocardial motion from cine DENSE images using spatiotemporal phase unwrapping and temporal fitting. IEEE Transactions on medical imaging, 26(1), 15-30.

Trabelsi O, Davis FM, Rodriguez-Matas JF, Duprey A, Avril S. 2015. Patient specific stress and rupture analysis of ascending thoracic aneurysms. Journal of Biomechanics, 48(10), 1836-1843.

Tustison NJ, Amini A. 2006. Biventricular myocardial strains via nonrigid registration of AnFigatomical NURBS models. Medical Imaging, IEEE Transactions on, 25(1), 94-112.

Usyk TP, Mazhari R, McCulloch AD. 2000. Effect of laminar orthotropic myofiber architecture on regional stress and strain in the canine left ventricle. Journal Of elasticity and the physical science of solids, 61(1-3):143--164.

Walker JC, Ratcliffe MB, Zhang P, Wallace AW, Fata B, Hsu EW, Guccioné JM. 2005. MRI-based finite-element analysis of left ventricular aneurysm. American Journal of Physiology-Heart and Circulatory Physiology, 289(2), H692-H700. 
Wenk JF, Wall ST, Peterson RC, Helgerson SL, Guccioné JM and et al.. 2009. A method for automatically optimizing medical devices for treating heart failure: designing polymeric injection patterns. Journal of Biomechanical Engineering , 131(12):121011.

Wang DH, Makaroun MS, Webster MW, Vorp DA. 2002. Effect of intraluminal thrombus on wall stress in patient-specific models of abdominal aortic aneurysm. Journal of Vascular Surgery, 36(3), 598-604.

Wang DH, Gao H, Luo XY, Berry C, Griffith BE, Ogden RW, Wang TJ. 2013. Structure-based finite strain modelling of the human left ventricle in diastole. International Journal for Numerical Methods in Biomedical Engineering,, 29(1):83--103.

Xi J, Lamata P, Lee J, Moireau P, Chapelle D, Smith N. 2011. Myocardial transversely isotropic material parameter estimation from in-silico measurements based on a reduced-order unscented kalman filter. Journal of the Mechanical Behavior of Biomedical Materials , 4(7) :1090-1102.

Yin FCP. 1985. Applications of the finite-element method to ventricular mechanics Critical reviews in Biomedical Engineering,. 12:311-342. 
Table 1. MPs intervals for isotropic material model from Marchesseau et al,(2013).

Table 2. The Fiber and Sheet stress results across the equatorial plane in LV model for the Guccione strain energy function at ED in $\mathrm{kPa}$. The stress values at the equatorial plane show higher average values in comparison to the isotopic stress results at the equatorial plane (Figure 4).

Table 3. The Fiber stress and strain results through wall thickness from epicardium to endocardium along two paths (L1 and L2) at ED. The stress and strain results are concentrated where the fibers are aligned with the circumferential direction. In inflation loading, the circumferential direction is naturally the direction of the major principal stress.

Table 4. Values of correlation coefficients for four MPs and Cauchy Fiber stress values $(\mathrm{kPa})$ at ED obtained with the Guccione material model after application of zero-pressure algorithm. This table shows a strong correlation of the fiber stress results to the linear coefficient before the exponential term of the strain energy function.

Table 5. Fiber stress across wall thickness from epicardium to endocardium along two paths

ES. 
Table 1

\begin{tabular}{ccc}
\hline \hline $\mathrm{c}_{1}(\mathrm{kPa})$ & $\mathrm{c}_{2}(\mathrm{kPa})$ & $\mathrm{d}_{1}(\mathrm{MPa})$ \\
\hline $7-20$ & $7-20$ & $0.08-0.3$ \\
\hline
\end{tabular}


Table 2

\begin{tabular}{|c|c|c|c|c|c|c|}
\hline & $\mathrm{c}(\mathrm{kPa})$ & $\mathrm{b}_{3}$ & $\mathrm{~b}_{2}$ & $\mathrm{~b}_{1}$ & $\begin{array}{c}\text { Mean Fiber } \\
\text { stress }\end{array}$ & $\begin{array}{c}\text { Mean Sheet } \\
\text { stress }\end{array}$ \\
\hline 1.Okamoto et al., 2000 & 0.512 & 67.1 & 24.2 & 21.6 & 4.4 & 2.1 \\
\hline 2.Keldermann et al., 2010 & 1.2 & 26.7 & 13.5 & 14.7 & 4 & 2.5 \\
\hline 3.Wenk et al., 2009 & 0.33 & 49.3 & 19.3 & 17.4 & 4.3 & 2.2 \\
\hline 4.Walker et al., 2005 & 0.128 & 53.7 & 21.3 & 17.3 & 4.2 & 2.4 \\
\hline 5.Walker et al., 2005 & 0.146 & 67.1 & 26.6 & 21.6 & 4.2 & 2.3 \\
\hline 6.Walker et al., 2005 & 0.233 & 49.3 & 19.3 & 17.4 & 4.3 & 2.3 \\
\hline 7.Xi et al., 2011 & 0.189 & 29.9 & 13.5 & 13.3 & 4.1 & 2.6 \\
\hline 8.Augenstein et al., 2005 & 3 & 11.1 & 1.8 & 10 & 5.4 & 1 \\
\hline 9.Land et al., 2012 & 1.662 & 14.3 & 4.5 & 10 & 4.6 & 1.8 \\
\hline 10.Omens et al., 1991 & 2.4 & 26.7 & 2 & 14.7 & 6.2 & 0.2 \\
\hline 11.Walker et al., 2005 & 0.359 & 67.1 & 24.2 & 21.6 & 4.4 & 2.1 \\
\hline 12.Omens et al., 1993 & 2.2 & 9.2 & 2 & 3.7 & 5.2 & 1.3 \\
\hline
\end{tabular}


Table3

Fiber stress $(\mathrm{kPa})$

\begin{tabular}{|c|c|c|c|c|c|c|c|c|c|c|c|}
\hline Mean & 2.58 & 3.16 & 3.77 & 4.35 & 4.75 & 4.91 & 4.77 & 4.34 & 3.73 & 3.01 & 2.48 \\
\hline${ }^{\mathrm{L} 1} \mathrm{SD}\left(10^{-3}\right)$ & 7 & 5 & 6 & 4 & 6 & 4 & 6 & 8 & 8 & 13 & 22 \\
\hline Mean & 1.61 & 1.96 & 2.45 & 2.99 & 3.47 & 3.8 & 3.89 & 3.7 & 3.24 & 2.61 & 1.75 \\
\hline${ }^{\mathrm{L} 2} \mathrm{SD}\left(10^{-3}\right)$ & 3 & 4 & 4 & 5 & 3 & 3 & 3 & 5 & 11 & 12 & 21 \\
\hline
\end{tabular}

Fiber strain

\begin{tabular}{cccccccccccc}
\hline Mean $\left(10^{-3}\right)$ & 5 & 10 & 15.4 & 20 & 23.9 & 25.1 & 23.6 & 19.6 & 13.7 & 7 & 1 \\
L1 SD $\left(10^{-3}\right)$ & 1 & 1 & 2 & 3 & 4 & 4 & 4 & 3 & 2 & 1 & 0 \\
\hline Mean $\left(10^{-3}\right)$ & 2.2 & 5 & 9 & 13 & 17 & 20 & 20 & 18 & 13 & 7 & 0 \\
L2 $\mathrm{SD}\left(10^{-3}\right)$ & 0.4 & 0.9 & 1 & 2 & 3 & 3 & 3 & 3 & 2 & 1 & 0 \\
\hline
\end{tabular}


Table 4

\begin{tabular}{ccc}
\hline \hline & Mean fiber stress & Mean sheet stress \\
\hline$c$ & 0.82 & -0.86 \\
$b_{3}$ & -0.5 & 0.52 \\
$b_{2}$ & -0.72 & 0.74 \\
$b_{1}$ & -0.44 & 0.45 \\
\hline
\end{tabular}




\section{Table 5}

Fiber stress (kPa)

\begin{tabular}{llccccccccccc}
\hline & Mean & 89.9 & 91.4 & 91.3 & 88.4 & 81.9 & 73.5 & 64.5 & 58.1 & 54.4 & 57 & 61.6 \\
L1 & SD & 6.4 & 6.2 & 5.5 & 4.3 & 3.1 & 2.9 & 3.5 & 4 & 4.1 & 4 & 3.8 \\
& & & & & & & & & & & & \\
\hline \multirow{2}{*}{$\begin{array}{l}\text { Mean } \\
\text { N }\end{array}$} & 35.1 & 40.6 & 46.2 & 54.2 & 67.3 & 82.2 & 90.1 & 80.2 & 58.7 & 32.7 & 9.5 \\
& 4.2 & 3.8 & 3.5 & 3.1 & 2.5 & 2.6 & 3.4 & 2.8 & 3 & 3.7 & 3.5 \\
\hline \hline
\end{tabular}


Figure 1. (a) LV model meshed in the Abaqus ${ }^{\circledR}$ software. (b) Selected paths across wall thickness used to study the stress variations across the thickness and the impact of MPs onto these stresses. The selected trajectories, L1 and L2, belong to the septal and lateral wall, respectively. In this case LV is divided into three equal segments longitudinally in order to study the evolution of stress in different LV level.

Figure 2. Personalized LV model with fiber structure. The red streamlines are aligned along fiber directions in the local coordinate system.

Figure 3. Flowchart of the zero-pressure algorithm used to obtain the load-free geometry. The image-based geometry was considered as the initial loaded configuration. Then, the algorithm returned the unloaded geometry of this initial configuration.

Figure 4. Mean and standard deviation of the fiber stress and strain for 40 isotropic and 12 anisotropic models.

Figure 5. Forward elastic simulation of the ED geometry using a linear elastic infinitesimal resolution with rigid parameters (10 MPa and 0.49 as elastic modulus and Poisson's ratio). The obtained results at ED (displacement and Von-Mises stress on the left hand side) are compared with the ones (right hand side) using the zero-pressure algorithm with the Mooney-Rivlin material model (for $c_{1}=0.0117, c_{2}=0.0182$ and $\left.d_{1}=0.1591\right)$. The displacements $\mathrm{U}$ are in $\mathrm{mm}$ and the Von-Mises stresses in MPa.

Figure 6. Reference ES geometry (green shaded) overlaid onto the ED initial configuration (grey shaded) in $3 \mathrm{D}$ representation and at different transversal cross sections. The simulation with active contraction produced an ejection fraction of $33.83 \%$ and a wall thickness change of $18.7 \%$.

Figure 7. Mean and standard deviation of Maximum-Principal and Von-Mises stress results reconstructed at ES taking into account the active contraction. The mean vonmises stresses increase from the pressurized endocardial surface to the epicardial surface. 
Figure 1

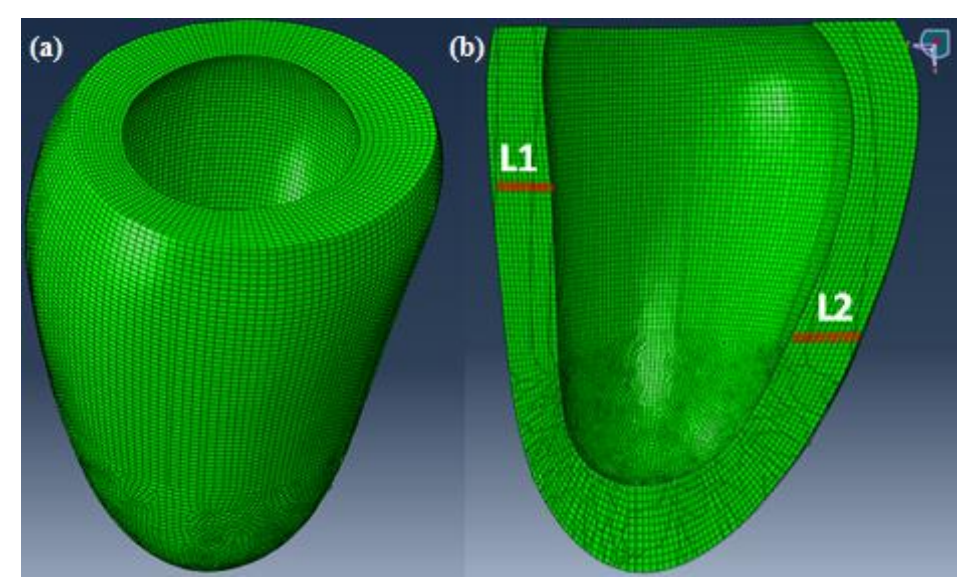


Figure 2

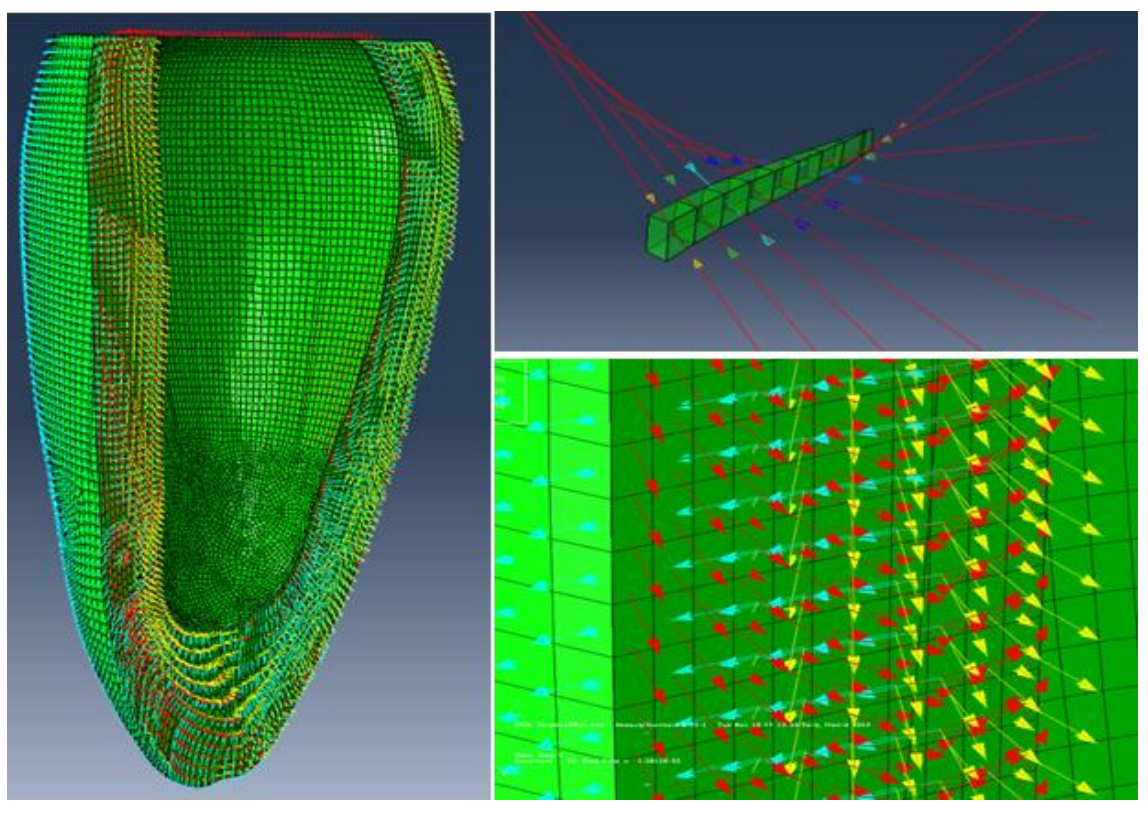


Figure 3

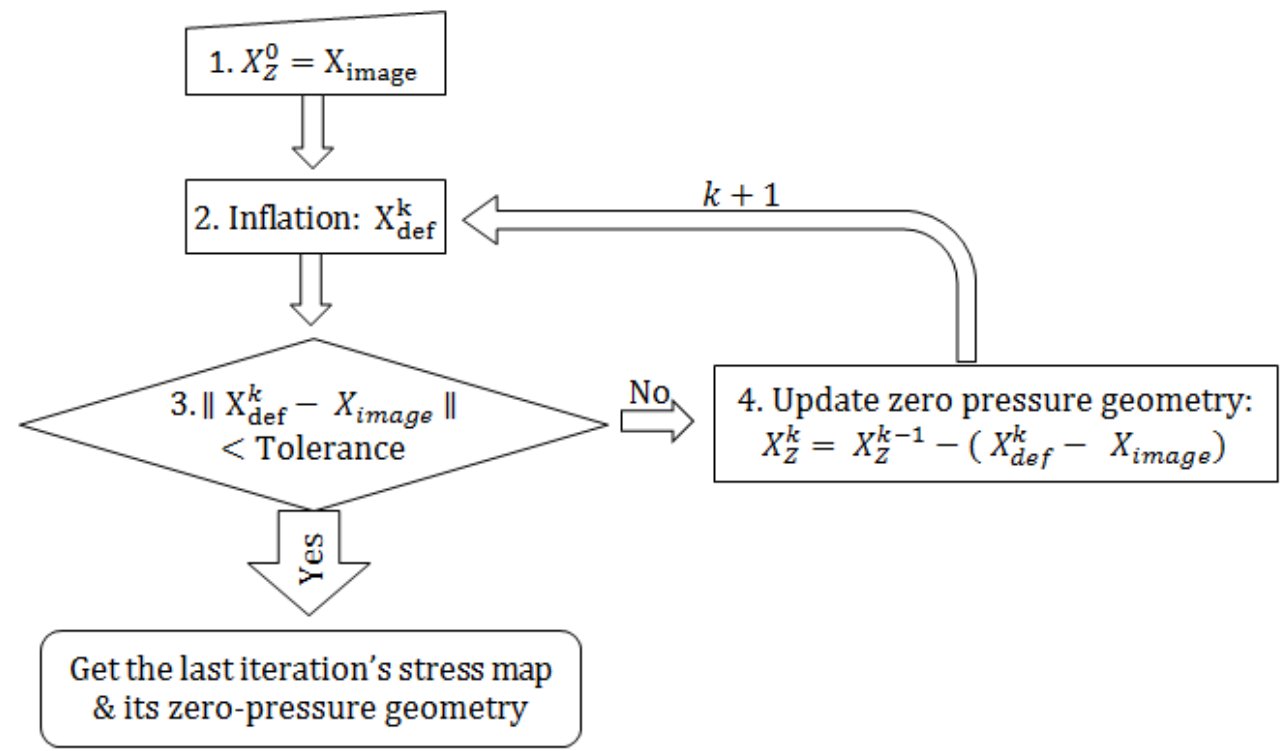


Figure 4 
Figure 5

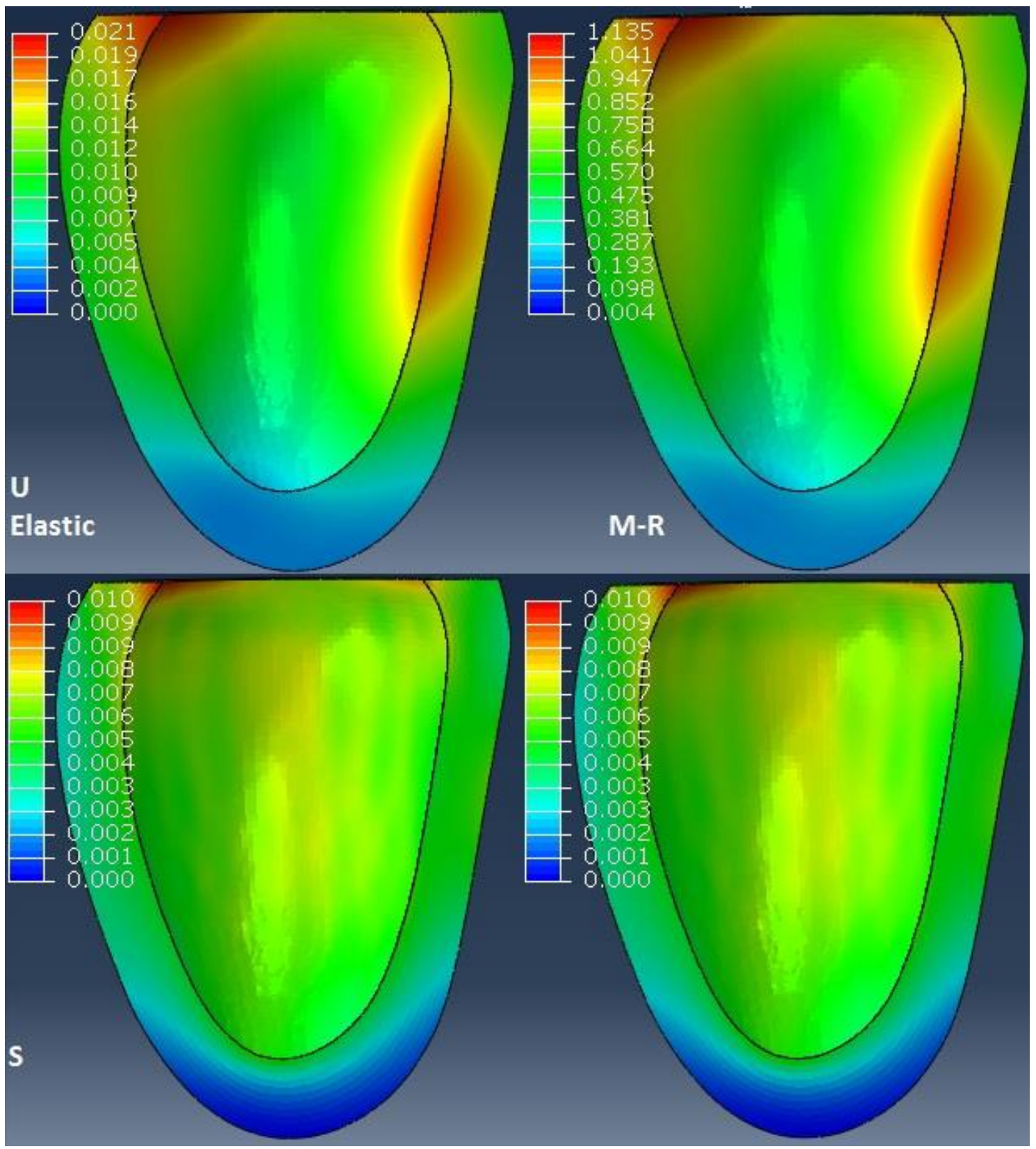


Figure 6

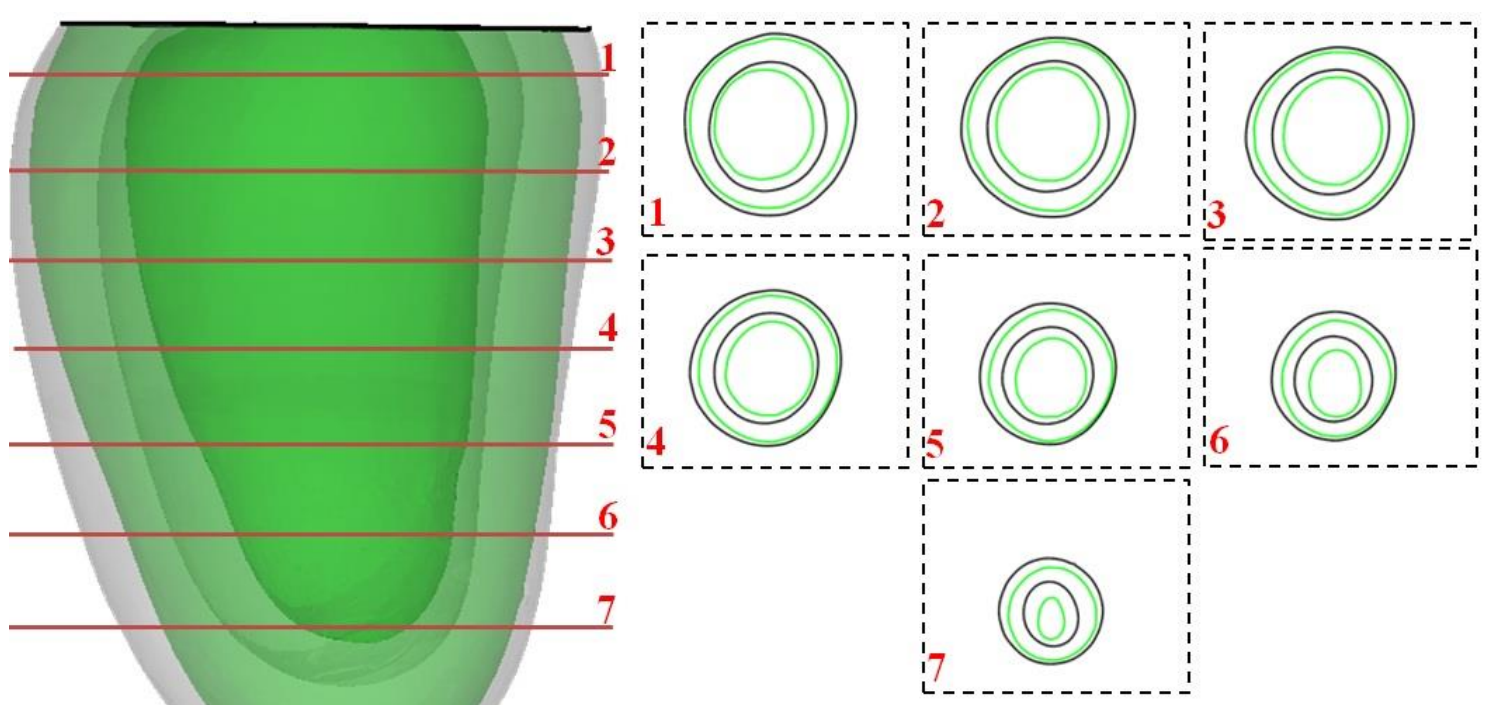


Figure 7

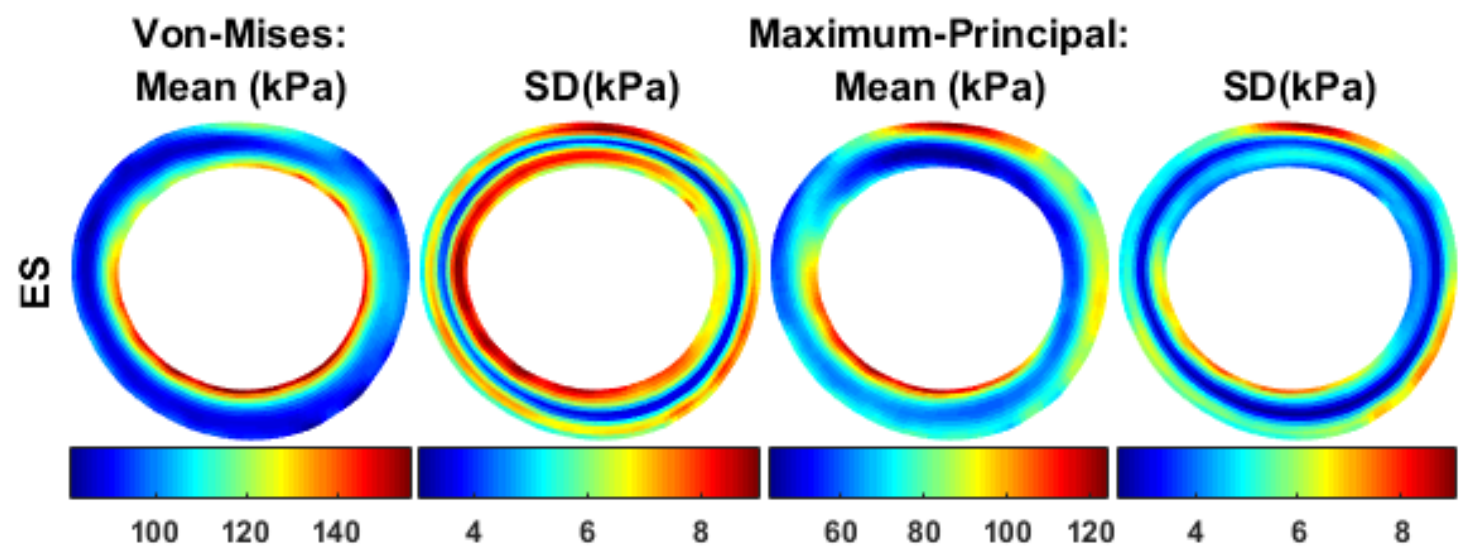

